\title{
New refinements of the discrete Jensen's inequality generated by finite or infinite permutations
}

\author{
LÁSZLÓ HoRVÁTH(1)
}

\begin{abstract}
In this paper some new refinements of the discrete Jensen's inequality are obtained in real vector spaces. The idea comes from some former refinements determined by cyclic permutations. We essentially generalize and extend these results by using permutations of finite sets and bijections of the set of positive numbers. We get refinements of the discrete Jensen's inequality for infinite convex combinations in Banach spaces. Similar results are rare. Finally, some applications are given on different topics.
\end{abstract}

Mathematics Subject Classification. Primary 26D15; Secondary 26B25.

Keywords. Discrete Jensen's inequality, Refinements, Infinite sums, Finite and infinite permutations.

\section{Introduction}

Different variants of Jensen's inequality and other inequalities have their origin in the notion of convexity. A real function $f$ defined on a convex subset $C$ of a real vector space is called convex if it satisfies

$$
f\left(\alpha v_{1}+(1-\alpha) v_{2}\right) \leq \alpha f\left(v_{1}\right)+(1-\alpha) f\left(v_{2}\right)
$$

for all $v_{1}, v_{2} \in C$ and all $\alpha \in[0,1]$.

The set of positive integers will be denoted by $\mathbb{N}_{+}$.

The following versions of Jensen's inequality are well known.

Theorem 1.1. (discrete Jensen's inequalities, see [11] and [13]) (a) Let $C$ be a convex subset of a real vector space $V$, and let $f: C \rightarrow \mathbb{R}$ be a convex function. If $p_{1}, \ldots, p_{n}$ are nonnegative numbers with $\sum_{i=1}^{n} p_{i}=1$, and $v_{1}, \ldots, v_{n} \in C$, then

$$
f\left(\sum_{i=1}^{n} p_{i} v_{i}\right) \leq \sum_{i=1}^{n} p_{i} f\left(v_{i}\right) .
$$


(b) Let $C$ be a closed convex subset of a real Banach space $V$, and let $f: C \rightarrow \mathbb{R}$ be a convex function. If $p_{1}, p_{2}, \ldots$ are nonnegative numbers with $\sum_{i=1}^{\infty} p_{i}=1$, and $v_{1}, v_{2}, \ldots \in C$ such that the series $\sum_{i=1}^{\infty} p_{i} v_{i}$ and $\sum_{i=1}^{\infty} p_{i} f\left(v_{i}\right)$ are absolutely convergent, then

$$
f\left(\sum_{i=1}^{\infty} p_{i} v_{i}\right) \leq \sum_{i=1}^{\infty} p_{i} f\left(v_{i}\right) .
$$

To give refinements of the discrete Jensen's inequality (1.1) is an extensively investigated theme with numerous methods and results (see e.g. the book [8] and references therein), and applications (see e.g. [5] and [6]). Then again, to the best of my knowledge, there are no refinements of the discrete Jensen's inequality (1.2) in such generality. There are some refinements of (1.2) when $C$ is an interval of $\mathbb{R}$ : either one estimates formulas in (1.2) in a suitable way (see [12]) or one can obtain results from refinements of integral Jensen's inequality (see $[7])$.

The following refinement of (1.1) can be found in [9] (see also [1]).

Theorem 1.2. Let $2 \leq k \leq n$ be integers, and let $p_{1}, \ldots, p_{n}$ and $\lambda_{1}, \ldots, \lambda_{k}$ be positive numbers with $\sum_{i=1}^{n} p_{i}=1$ and $\sum_{i=1}^{k} \lambda_{i}=1$. If $C$ is a convex subset of a real vector space $V, f: C \rightarrow \mathbb{R}$ is a convex function, and $v_{1}, \ldots, v_{n} \in C$, then

$$
\begin{aligned}
f\left(\sum_{i=1}^{n} p_{i} v_{i}\right) & \leq C_{d i s}=C_{d i s}(f, \mathbf{v}, \mathbf{p}, \boldsymbol{\lambda}) \\
& :=\sum_{i=1}^{n}\left(\sum_{j=0}^{k-1} \lambda_{j+1} p_{i+j}\right) f\left(\frac{\sum_{j=0}^{k-1} \lambda_{j+1} p_{i+j} v_{i+j}}{\sum_{j=0}^{k-1} \lambda_{j+1} p_{i+j}}\right) \\
& \leq \sum_{i=1}^{n} p_{i} f\left(v_{i}\right),
\end{aligned}
$$

where $i+j$ means $i+j-n$ in case of $i+j>n$.

It is easy to think that the previous result cannot be generalized for infinite sums, but we can observe that the middle term $C_{d i s}$ in (1.3) can be rewritten in the following form

$$
C_{d i s}=\sum_{i=1}^{n}\left(\sum_{j=1}^{k} \lambda_{j} p_{\pi_{j}(i)}\right) f\left(\frac{\sum_{j=1}^{k} \lambda_{j} p_{\pi_{j}(i)} v_{\pi_{j}(i)}}{\sum_{j=1}^{k} \lambda_{j} p_{\pi_{j}(i)}}\right),
$$

where $\pi_{j}(j=1, \ldots, k)$ is the $(j-1)$-cyclic permutation of the set $\{1, \ldots, n\}$ to the right (all elements are moved to the right $j-1$ times with elements overflowing from the right being inserted to the left).

In this paper we show that formulas like (1.4) refine both (1.1) and (1.2) by using either permutations of the set $\{1, \ldots, n\}$ or bijections from $\mathbb{N}_{+}$onto 
itself. On the one hand, an essential generalization of Theorem 1.2 is given, on the other hand, refinements of (1.2) are developed without assuming that $V$ is a special Banach space. Finally, we give some applications concerning information theory, the norm function, Hölder's inequality and the inequality of arithmetic and geometric means.

\section{Main results}

The positive part $f^{+}$and the negative part $f^{-}$of a real valued function $f$ are defined in the usual way.

Let the set $I$ denote either $\{1, \ldots, n\}$ for some $n \geq 1$ or $\mathbb{N}_{+}$. We say that the numbers $\left(p_{i}\right)_{i \in I}$ represent a (positive) discrete probability distribution if $\left(p_{i}>0\right) p_{i} \geq 0(i \in I)$ and $\sum_{i \in I} p_{i}=1$. A permutation $\pi$ of $I$ refers to a bijection from $I$ onto itself.

We need the following hypotheses which are partitioned into two classes:

$\left(\mathrm{H}_{1}\right)$ Let $k, n \geq 2$ be integers, and let $p_{1}, \ldots, p_{n}$ and $\lambda_{1}, \ldots, \lambda_{k}$ represent positive probability distributions.

$\left(\mathrm{H}_{2}\right)$ For each $j=1, \ldots, k$ let $\pi_{j}$ be a permutation of the set $\{1, \ldots, n\}$.

$\left(\mathrm{H}_{3}\right)$ Let $C$ be a convex subset of a real vector space $V$, and $f: C \rightarrow \mathbb{R}$ be a convex function.

$\left(\mathrm{C}_{1}\right)$ Let the set $J$ denote either $\{1, \ldots, k\}$ for some $k \geq 2$ or $\mathbb{N}_{+}$. Let $p_{1}, p_{2}, \ldots$ and $\left(\lambda_{j}\right)_{j \in J}$ represent positive probability distributions.

$\left(\mathrm{C}_{2}\right)$ For each $j \in J$ let $\pi_{j}$ be a permutation of the set $\mathbb{N}_{+}$.

$\left(\mathrm{C}_{3}\right)$ Let $C$ be a closed convex subset of a real Banach space $(V,\|\cdot\|)$, and $f: C \rightarrow \mathbb{R}$ be a convex function.

Theorem 2.1. (a) Assume $\left(H_{1}\right),\left(H_{2}\right)$ and $\left(H_{3}\right)$. If $v_{1}, \ldots, v_{n} \in C$, then

$$
\begin{aligned}
f\left(\sum_{i=1}^{n} p_{i} v_{i}\right) & \leq C_{\text {per }}=C_{\text {per }}(f, \mathbf{v}, \mathbf{p}, \boldsymbol{\lambda}, \boldsymbol{\pi}) \\
& :=\sum_{i=1}^{n}\left(\sum_{j=1}^{k} \lambda_{j} p_{\pi_{j}(i)}\right) f\left(\frac{\sum_{j=1}^{k} \lambda_{j} p_{\pi_{j}(i)} v_{\pi_{j}(i)}}{\sum_{j=1}^{k} \lambda_{j} p_{\pi_{j}(i)}}\right) \\
& \leq \sum_{i=1}^{n} p_{i} f\left(v_{i}\right) .
\end{aligned}
$$

(b) Assume $\left(C_{1}\right),\left(C_{2}\right)$ and $\left(C_{3}\right)$. If $v_{1}, v_{2}, \ldots \in C$ such that the series $\sum_{i=1}^{\infty} p_{i} v_{i}$ and $\sum_{i=1}^{\infty} p_{i} f\left(v_{i}\right)$ are absolutely convergent, then

$$
f\left(\sum_{i=1}^{\infty} p_{i} v_{i}\right) \leq C_{p e r}=C_{p e r}(f, \mathbf{v}, \mathbf{p}, \boldsymbol{\lambda}, \boldsymbol{\pi})
$$




$$
\begin{aligned}
: & =\sum_{i=1}^{\infty}\left(\sum_{j \in J} \lambda_{j} p_{\pi_{j}(i)}\right) f\left(\frac{\sum_{j \in J} \lambda_{j} p_{\pi_{j}(i)} v_{\pi_{j}(i)}}{\sum_{j \in J} \lambda_{j} p_{\pi_{j}(i)}}\right) \\
& \leq \sum_{i=1}^{\infty} p_{i} f\left(v_{i}\right) .
\end{aligned}
$$

Proof. (a) By using Theorem 1.1 (a) and the fact that $\pi_{j}$ is a permutation of the set $\{1, \ldots, n\}$,

$$
\begin{aligned}
C_{\text {per }} & \leq \sum_{i=1}^{n}\left(\sum_{j=1}^{k} \lambda_{j} p_{\pi_{j}(i)} f\left(v_{\pi_{j}(i)}\right)\right)=\sum_{j=1}^{k} \lambda_{j}\left(\sum_{i=1}^{n} p_{\pi_{j}(i)} f\left(v_{\pi_{j}(i)}\right)\right) \\
& =\left(\sum_{j=1}^{k} \lambda_{j}\right)\left(\sum_{i=1}^{n} p_{i} f\left(v_{i}\right)\right)=\sum_{i=1}^{n} p_{i} f\left(v_{i}\right) .
\end{aligned}
$$

The left hand side inequality can be proved similarly. Since

$$
\sum_{i=1}^{n}\left(\sum_{j=1}^{k} \lambda_{j} p_{\pi_{j}(i)}\right)=\left(\sum_{j=1}^{k} \lambda_{j}\right)\left(\sum_{i=1}^{n} p_{i}\right)=1,
$$

the discrete Jensen's inequality implies that

$$
C_{p e r} \geq f\left(\sum_{i=1}^{n}\left(\sum_{j=1}^{k} \lambda_{j} p_{\pi_{j}(i)} v_{\pi_{j}(i)}\right)\right)=f\left(\sum_{i=1}^{n} p_{i} v_{i}\right) .
$$

(b) The proof is divided into four parts.

I. We first prove that the series

$$
\sum_{i=1}^{\infty}\left(\sum_{j \in J} \lambda_{j} p_{\pi_{j}(i)}\left\|v_{\pi_{j}(i)}\right\|\right)
$$

and

$$
\sum_{i=1}^{\infty}\left(\sum_{j \in J} \lambda_{j} p_{\pi_{j}(i)}\left|f\left(v_{\pi_{j}(i)}\right)\right|\right)
$$

are convergent and

$$
\sum_{i=1}^{\infty}\left(\sum_{j \in J} \lambda_{j} p_{\pi_{j}(i)} v_{\pi_{j}(i)}\right)=\sum_{i=1}^{\infty} p_{i} v_{i}
$$

and

$$
\sum_{i=1}^{\infty}\left(\sum_{j \in J} \lambda_{j} p_{\pi_{j}(i)} f\left(v_{\pi_{j}(i)}\right)\right)=\sum_{i=1}^{\infty} p_{i} f\left(v_{i}\right)
$$


For each $j \in J$ the series

$$
\sum_{i=1}^{\infty} p_{\pi_{j}(i)} v_{\pi_{j}(i)}
$$

is a rearrangement of the absolutely convergent series $\sum_{i=1}^{\infty} p_{i} v_{i}$, and hence it is also absolutely convergent and

$$
\sum_{i=1}^{\infty} p_{\pi_{j}(i)} v_{\pi_{j}(i)}=\sum_{i=1}^{\infty} p_{i} v_{i} .
$$

(i) If $J=\{1, \ldots, k\}$, then it follows trivially from (2.7) that

$$
\sum_{i=1}^{\infty}\left(\sum_{j=1}^{k} \lambda_{j} p_{\pi_{j}(i)} v_{\pi_{j}(i)}\right)=\sum_{i=1}^{\infty} p_{i} v_{i}
$$

(ii) Assume $J=\mathbb{N}_{+}$. The property of absolute convergence of (2.6) implies that

$$
\begin{aligned}
\sum_{j=1}^{\infty}\left(\sum_{i=1}^{\infty} \lambda_{j} p_{\pi_{j}(i)}\left\|v_{\pi_{j}(i)}\right\|\right) & =\sum_{j=1}^{\infty} \lambda_{j}\left(\sum_{i=1}^{\infty} p_{\pi_{j}(i)}\left\|v_{\pi_{j}(i)}\right\|\right) \\
& =\sum_{i=1}^{\infty} p_{i}\left\|v_{i}\right\|<\infty .
\end{aligned}
$$

Therefore, as it is well known, the order of summation can be interchanged in the double sum

$$
\sum_{i=1}^{\infty}\left(\sum_{j=1}^{\infty} \lambda_{j} p_{\pi_{j}(i)} f\left(v_{\pi_{j}(i)}\right)\right)
$$

and hence

$$
\sum_{i=1}^{\infty}\left(\sum_{j=1}^{\infty} \lambda_{j} p_{\pi_{j}(i)} f\left(v_{\pi_{j}(i)}\right)\right)=\sum_{j=1}^{\infty}\left(\sum_{i=1}^{\infty} \lambda_{j} p_{\pi_{j}(i)} v_{\pi_{j}(i)}\right)=\sum_{i=1}^{\infty} p_{i} v_{i} .
$$

The series in (2.4) can be handled in a similar way.

II. (i) If $J=\{1, \ldots, k\}$, then Theorem 1.1 (a) gives us that for every $n \in \mathbb{N}_{+}$

$$
\sum_{i=1}^{n}\left(\sum_{j=1}^{k} \lambda_{j} p_{\pi_{j}(i)}\right) f\left(\frac{\sum_{j=1}^{k} \lambda_{j} p_{\pi_{j}(i)} v_{\pi_{j}(i)}}{\sum_{j=1}^{k} \lambda_{j} p_{\pi_{j}(i)}}\right) \leq \sum_{i=1}^{n}\left(\sum_{j=1}^{k} \lambda_{j} p_{\pi_{j}(i)} f\left(v_{\pi_{j}(i)}\right)\right) .
$$

(ii) Assume $J=\mathbb{N}_{+}$. For each $i \in \mathbb{N}_{+}$the series $\sum_{j=1}^{\infty} \lambda_{j} p_{\pi_{j}(i)}$ is obviously convergent and

$$
\frac{\sum_{j=1}^{\infty} \lambda_{j} p_{\pi_{j}(i)}\left\|v_{\pi_{j}(i)}\right\|}{\sum_{j=1}^{\infty} \lambda_{j} p_{\pi_{j}(i)}} \leq \frac{1}{\sum_{j=1}^{\infty} \lambda_{j} p_{\pi_{j}(i)}} \sum_{j=1}^{\infty} p_{\pi_{j}(i)}\left\|v_{\pi_{j}(i)}\right\|
$$




$$
=\frac{1}{\sum_{j=1}^{\infty} \lambda_{j} p_{\pi_{j}(i)}} \sum_{i=1}^{\infty} p_{i}\left\|v_{i}\right\|<\infty,
$$

and hence the series

$$
\frac{\sum_{j=1}^{\infty} \lambda_{j} p_{\pi_{j}(i)}\left\|v_{\pi_{j}(i)}\right\|}{\sum_{j=1}^{\infty} \lambda_{j} p_{\pi_{j}(i)}}
$$

is absolutely convergent. Further, we know from part I that the series

$$
\sum_{j=1}^{\infty} \lambda_{j} p_{\pi_{j}(i)} f\left(v_{\pi_{j}(i)}\right), \quad i \in \mathbb{N}_{+}
$$

is also absolutely convergent.

Consequently, Theorem 1.1 (b) shows that

$$
\sum_{i=1}^{n}\left(\sum_{j=1}^{\infty} \lambda_{j} p_{\pi_{j}(i)}\right) f\left(\frac{\sum_{j=1}^{\infty} \lambda_{j} p_{\pi_{j}(i)} v_{\pi_{j}(i)}}{\sum_{j=1}^{\infty} \lambda_{j} p_{\pi_{j}(i)}}\right) \leq \sum_{i=1}^{n}\left(\sum_{j=1}^{\infty} \lambda_{j} p_{\pi_{j}(i)} f\left(v_{\pi_{j}(i)}\right)\right) .
$$

We have seen in part I that the series (2.4) is convergent and its sum is $\sum_{i=1}^{\infty} p_{i} f\left(v_{i}\right)$. Thus by (2.8) or by (2.9), the second inequality in (2.2) will be proved if we succeed in showing that the series

$$
\sum_{i=1}^{\infty}\left(\sum_{j \in J} \lambda_{j} p_{\pi_{j}(i)}\right) f\left(\frac{\sum_{j \in J} \lambda_{j} p_{\pi_{j}(i)} v_{\pi_{j}(i)}}{\sum_{j \in J} \lambda_{j} p_{\pi_{j}(i)}}\right)
$$

is convergent.

It is known that the positive part of $f$ is also convex. The convergence of (2.4) implies the convergence of the series

$$
\sum_{i=1}^{\infty}\left(\sum_{j \in J} \lambda_{j} p_{\pi_{j}(i)} f^{+}\left(v_{\pi_{j}(i)}\right)\right) .
$$

It now follows that we can copy the proofs of (2.8) and (2.9) with $f^{+}$instead of $f$. Taking account of the nonnegativity of $f^{+}$, we obtain

$$
\begin{gathered}
\sum_{i=1}^{\infty}\left(\sum_{j \in J} \lambda_{j} p_{\pi_{j}(i)}\right) f^{+}\left(\frac{\sum_{j \in J} \lambda_{j} p_{\pi_{j}(i)} v_{\pi_{j}(i)}}{\sum_{j \in J} \lambda_{j} p_{\pi_{j}(i)}}\right) \\
\leq \sum_{i=1}^{\infty}\left(\sum_{j \in J} \lambda_{j} p_{\pi_{j}(i)} f^{+}\left(v_{\pi_{j}(i)}\right)\right)<\infty .
\end{gathered}
$$


From this it follows that the series (2.10) will be convergent (absolutely) if and only if

$$
\sum_{i=1}^{\infty}\left(\sum_{j \in J} \lambda_{j} p_{\pi_{j}(i)}\right) f^{-}\left(\frac{\sum_{j \in J} \lambda_{j} p_{\pi_{j}(i)} v_{\pi_{j}(i)}}{\sum_{j \in J} \lambda_{j} p_{\pi_{j}(i)}}\right)<\infty .
$$

III. In this step we show that the series (2.12) is convergent and the first inequality in (2.2) holds assuming $f$ is bounded below, that is $f(v) \geq c(v \in C)$ for some nonpositive number $c$.

Since

$$
\begin{aligned}
& \sum_{i=1}^{\infty}\left(\sum_{j \in J} \lambda_{j} p_{\pi_{j}(i)}\right) f^{-}\left(\frac{\sum_{j \in J} \lambda_{j} p_{\pi_{j}(i)} v_{\pi_{j}(i)}}{\sum_{j \in J} \lambda_{j} p_{\pi_{j}(i)}}\right) \leq-c \sum_{i=1}^{\infty}\left(\sum_{j \in J} \lambda_{j} p_{\pi_{j}(i)}\right) \\
& \quad=-c \sum_{j \in J} \lambda_{j}\left(\sum_{i=1}^{\infty} p_{\pi_{j}(i)}\right)=-c
\end{aligned}
$$

the series (2.12) is convergent.

According to (2.11) and (2.12) the series (2.10) is absolutely convergent.

Since the series (2.3) and (2.10) are absolutely convergent and (2.5) holds, we can apply Theorem 1.1 (b), and obtain the first inequality in (2.2).

IV. At this point we abandon the lower boundedness hypothesis on $f$.

Let the function $f_{n}: C \rightarrow \mathbb{R}$ be defined by

$$
f_{n}(v)=\max (f(v),-n), \quad n \in \mathbb{N}_{+} .
$$

Then $f_{n}\left(n \in \mathbb{N}_{+}\right)$is convex and bounded below, and $f_{n} \geq f\left(n \in \mathbb{N}_{+}\right)$. From this and from the results of part III, we get that for each $n \in \mathbb{N}_{+}$the series

$$
\sum_{i=1}^{\infty}\left(\sum_{j \in J} \lambda_{j} p_{\pi_{j}(i)}\right) f_{n}\left(\frac{\sum_{j \in J} \lambda_{j} p_{\pi_{j}(i)} v_{\pi_{j}(i)}}{\sum_{j \in J} \lambda_{j} p_{\pi_{j}(i)}}\right)
$$

is absolutely convergent and

$$
f\left(\sum_{i=1}^{\infty} p_{i} v_{i}\right) \leq f_{n}\left(\sum_{i=1}^{\infty} p_{i} v_{i}\right) \leq \sum_{i=1}^{\infty}\left(\sum_{j \in J} \lambda_{j} p_{\pi_{j}(i)}\right) f_{n}\left(\frac{\sum_{j \in J} \lambda_{j} p_{\pi_{j}(i)} v_{\pi_{j}(i)}}{\sum_{j \in J} \lambda_{j} p_{\pi_{j}(i)}}\right) .
$$

Since the sequence $\left(f_{n}\right)_{n \in \mathbb{N}_{+}}$is decreasing and $\lim _{n \rightarrow \infty} f_{n}=f$ pointwise, the previous two assertions imply that B. Levi's theorem can be applied, and it gives that the series (2.10) is absolutely convergent and the first inequality in (2.2) holds.

The proof is complete.

Remark 2.2. It can be seen that Theorem 2.1 (a) contains Theorem 1.2 as a special case. 


\section{Applications}

We begin with some inequalities corresponding to information theory.

The following notion was introduced by Csiszár in [2] and [3].

Definition 3.1. Let $f:] 0, \infty[\rightarrow] 0, \infty[$ be a convex function, and let $\mathbf{r}:=$ $\left.\left(r_{1}, \ldots, r_{n}\right) \in\right] 0, \infty\left[^{n}\right.$ and $\left.\mathbf{q}:=\left(q_{1}, \ldots, q_{n}\right) \in\right] 0, \infty\left[{ }^{n}\right.$. The $f$-divergence functional is

$$
I_{f}(\mathbf{r}, \mathbf{q}):=\sum_{i=1}^{n} q_{i} f\left(\frac{r_{i}}{q_{i}}\right) .
$$

Based on this concept, we have introduced a new functional in [10], and this functional can be further generalized:

Definition 3.2. Let $C$ be a convex subset of a real vector space $V$, and $f: C \rightarrow$ $\mathbb{R}$ be a convex function. If $\mathbf{w}:=\left(w_{1}, \ldots, w_{n}\right) \in V^{n}$ and $\mathbf{q}:=\left(q_{1}, \ldots, q_{n}\right) \in$ ] $0, \infty\left[{ }^{n}\right.$ such that

$$
\frac{w_{i}}{q_{i}} \in C, \quad i=1, \ldots, n,
$$

then define

$$
I_{f}(\mathbf{w}, \mathbf{q}):=\sum_{i=1}^{n} q_{i} f\left(\frac{w_{i}}{q_{i}}\right) .
$$

Proposition 3.3. Let $k, n \geq 2$ be integers, and let $\lambda_{1}, \ldots, \lambda_{k}$ represent a positive probability distribution. Assume $\left(H_{2}\right)$ and $\left(H_{3}\right)$. If $\mathbf{w}:=\left(w_{1}, \ldots, w_{n}\right) \in V^{n}$ and $\left.\mathbf{q}:=\left(q_{1}, \ldots, q_{n}\right) \in\right] 0, \infty\left[^{n}\right.$ such that

$$
\frac{w_{i}}{q_{i}} \in C, \quad i=1, \ldots, n,
$$

then

$$
\begin{aligned}
I_{f}(\mathbf{w}, \mathbf{q}) & =\sum_{i=1}^{n} q_{i} f\left(\frac{w_{i}}{q_{i}}\right) \\
& \geq \sum_{i=1}^{n}\left(\sum_{j=1}^{k} \lambda_{j} q_{\pi_{j}(i)}\right) f\left(\frac{\sum_{j=1}^{k} \lambda_{j} w_{\pi_{j}(i)}}{\sum_{j=1}^{k} \lambda_{j} q_{\pi_{j}(i)}}\right) \\
& \geq\left(\sum_{i=1}^{n} q_{i}\right) \cdot f\left(\frac{\sum_{i=1}^{n} w_{i}}{\sum_{i=1}^{n} q_{i}}\right) .
\end{aligned}
$$

Proof. By applying Theorem 2.1 (a) with

$$
p_{i}:=\frac{q_{i}}{\sum_{i=1}^{n} q_{i}}, \quad v_{i}:=\frac{w_{i}}{q_{i}}, \quad i=1, \ldots, n
$$


we have

$$
\begin{aligned}
\sum_{i=1}^{n} q_{i} f\left(\frac{w_{i}}{q_{i}}\right) & =\left(\sum_{i=1}^{n} q_{i}\right) \cdot \sum_{i=1}^{n} \frac{q_{i}}{\sum_{i=1}^{n} q_{i}} f\left(\frac{w_{i}}{q_{i}}\right) \\
& \geq\left(\sum_{i=1}^{n} q_{i}\right) \cdot \sum_{i=1}^{n}\left(\sum_{j=1}^{k} \lambda_{j} \frac{q_{\pi_{j}(i)}}{\sum_{i=1}^{n} q_{i}}\right) f\left(\frac{\sum_{j=1}^{k} \lambda_{j} \frac{q_{\pi_{j}(i)}}{\sum_{i=1}^{n} \frac{w_{\pi_{j}(i)}}{q_{\pi_{j}(i)}}}}{\sum_{j=1}^{k} \lambda_{j} \frac{q_{\pi_{j}(i)}}{\sum_{i=1}^{n} q_{i}}}\right) \\
& =\sum_{i=1}^{n}\left(\sum_{j=1}^{k} \lambda_{j} q_{\pi_{j}(i)}\right) f\left(\frac{\sum_{j=1}^{k} \lambda_{j} w_{\pi_{j}(i)}}{\sum_{j=1}^{k} \lambda_{j} q_{\pi_{j}(i)}}\right) \\
& \geq\left(\sum_{i=1}^{n} q_{i}\right) \cdot f\left(\frac{\sum_{i=1}^{n} w_{i}}{\sum_{i=1}^{n} q_{i}}\right) .
\end{aligned}
$$

The proof is complete.

Remark 3.4. (a) It was proved in [4] that if $f:] 0, \infty[\rightarrow] 0, \infty[$ is a convex function, and $\left.\mathbf{r}:=\left(r_{1}, \ldots, r_{n}\right) \in\right] 0, \infty\left[{ }^{n}\right.$ and $\left.\mathbf{q}:=\left(q_{1}, \ldots, q_{n}\right) \in\right] 0, \infty\left[^{n}\right.$, then

$$
I_{f}(\mathbf{r}, \mathbf{q}) \geq \sum_{i=1}^{n} q_{i} f\left(\frac{\sum_{i=1}^{n} r_{i}}{\sum_{i=1}^{n} q_{i}}\right)
$$

From Proposition 3.3 we can obtain the following refinement of this inequality:

$$
I_{f}(\mathbf{r}, \mathbf{q}) \geq \sum_{i=1}^{n}\left(\sum_{j=1}^{k} \lambda_{j} q_{\pi_{j}(i)}\right) f\left(\frac{\sum_{j=1}^{k} \lambda_{j} r_{\pi_{j}(i)}}{\sum_{j=1}^{k} \lambda_{j} q_{\pi_{j}(i)}}\right) \geq \sum_{i=1}^{n} q_{i} f\left(\frac{\sum_{i=1}^{n} r_{i}}{\sum_{i=1}^{n} q_{i}}\right) .
$$

(b) Let $f:=-\log$, where the base of $\log$ is greater than $1, \mathbf{r}:=(1, \ldots, 1)$, and $\mathbf{q}:=\left(q_{1}, \ldots, q_{n}\right)$ represent a positive probability distribution. Then (3.3) gives 


$$
\begin{aligned}
H(\mathbf{q}): & =-\sum_{i=1}^{n} q_{i} \log \left(q_{i}\right) \\
& \leq-\sum_{i=1}^{n}\left(\sum_{j=1}^{k} \lambda_{j} q_{\pi_{j}(i)}\right) \log \left(\sum_{j=1}^{k} \lambda_{j} q_{\pi_{j}(i)}\right) \leq \log (n),
\end{aligned}
$$

which is a refinement of a remarkable inequality for the Shannon entropy.

Next we establish inequalities for the norm function.

Proposition 3.5. Assume $\left(C_{1}\right)$ and $\left(C_{2}\right)$, and assume $(V,\|\cdot\|)$ is a Banach space. If $v_{1}, v_{2}, \ldots \in V$ such that the series $\sum_{i=1}^{\infty} p_{i}\left\|v_{i}\right\|^{\alpha}$ is absolutely convergent for some $\alpha \geq 1$, then

$$
\left\|\sum_{i=1}^{\infty} p_{i} v_{i}\right\|^{\alpha} \leq \sum_{i=1}^{\infty}\left(\sum_{j \in J} \lambda_{j} p_{\pi_{j}(i)}\right)\left\|\frac{\sum_{j \in J} \lambda_{j} p_{\pi_{j}(i)} v_{\pi_{j}(i)}}{\sum_{j \in J} \lambda_{j} p_{\pi_{j}(i)}}\right\|^{\alpha} \leq \sum_{i=1}^{\infty} p_{i}\left\|v_{i}\right\|^{\alpha} .
$$

Proof. Since the function $f: V \rightarrow \mathbb{R}$ defined by $f(v)=\|v\|^{\alpha}$ is convex, and the series $\sum_{i=1}^{\infty} p_{i} v_{i}$ is also absolutely convergent, the result follows from Theorem 2.1 (b).

Now we get a refinement of the discrete Hölder's inequality.

Proposition 3.6. Let the set $J$ denote either $\{1, \ldots, k\}$ for some $k \geq 2$ or $\mathbb{N}_{+}$, and let $\left(\lambda_{j}\right)_{j \in J}$ represent a positive probability distribution. Let $\left(w_{i}\right)_{i=1}^{\infty}$ be a sequence of positive numbers, and let $\left(x_{i}\right)_{i=1}^{\infty}$ and $\left(y_{i}\right)_{i=1}^{\infty}$ be sequences of nonnegative numbers such that the series $\sum_{i=1}^{\infty} w_{i} x_{i}^{p}$ and $\sum_{i=1}^{\infty} w_{i} y_{i}^{q}$ are convergent, where $p>1$ and $q>1$ are conjugate exponents that $i s \frac{1}{p}+\frac{1}{q}=1$. Then

$$
\begin{aligned}
\sum_{i=1}^{\infty} w_{i} x_{i} y_{i} & \leq \sum_{i=1}^{\infty}\left(\sum_{j \in J} \lambda_{j} w_{\pi_{j}(i)} x_{\pi_{j}(i)}^{p}\right)^{\frac{1}{p}}\left(\sum_{j \in J} \lambda_{j} w_{\pi_{j}(i)} y_{\pi_{j}(i)}^{q}\right)^{\frac{1}{q}} \\
& \leq\left(\sum_{i=1}^{\infty} w_{i} x_{i}^{p}\right)^{\frac{1}{p}}\left(\sum_{i=1}^{\infty} w_{i} y_{i}^{q}\right)^{\frac{1}{q}} .
\end{aligned}
$$

Proof. For each $s>1$ the power function

$$
\left.f_{s}:\right] 0, \infty\left[\rightarrow \mathbb{R}, \quad f_{s}(x)=x^{s}\right.
$$

is strictly convex. Let $v_{1}, v_{2} \ldots$ be positive numbers such that the series $\sum_{i=1}^{\infty}$ $p_{i} v_{i}^{s}$ is convergent. Theorem 2.1 (b) can be applied to the function $f_{s}$ and to the positive numbers $v_{1}, v_{2} \ldots$, and it yields that 


$$
\left(\sum_{i=1}^{\infty} p_{i} v_{i}\right)^{s} \leq \sum_{i=1}^{\infty}\left(\sum_{j \in J} \lambda_{j} p_{\pi_{j}(i)}\right)^{1-s}\left(\sum_{j \in J} \lambda_{j} p_{\pi_{j}(i)} v_{\pi_{j}(i)}\right)^{s} \leq \sum_{i=1}^{\infty} p_{i} v_{i}^{s} .
$$

If $\sum_{i=1}^{\infty} w_{i} y_{i}^{q}=0$, then (3.4) is obvious.

Otherwise, from the inequality (3.6) with the choices

$$
s=\frac{1}{p}, \quad p_{i}=\frac{w_{i} y_{i}^{q}}{\sum_{l=1}^{\infty} w_{l} y_{l}^{q}}, \quad v_{i}=x_{i}^{p} y_{i}^{-q}, \quad i \in \mathbb{N}_{+}
$$

$\left(-f_{1 / p}\right.$ is convex $)$ we obtain

$$
\begin{aligned}
& \left(\frac{1}{\sum_{l=1}^{\infty} w_{l} y_{l}^{q}}\right)^{\frac{1}{p}}\left(\sum_{i=1}^{\infty} w_{i} x_{i}^{p}\right)^{\frac{1}{p}} \geq \frac{1}{\sum_{l=1}^{\infty} w_{l} y_{l}^{q}} \\
& \cdot \sum_{i=1}^{\infty}\left(\sum_{j \in J} \lambda_{j} w_{\pi_{j}(i)} y_{\pi_{j}(i)}^{q}\right)^{\frac{1}{q}}\left(\sum_{j \in J} \lambda_{j} w_{\pi_{j}(i)} x_{\pi_{j}(i)}^{p}\right)^{\frac{1}{p}} \\
& \geq \frac{1}{\sum_{l=1}^{\infty} w_{l} y_{l}^{q}} \sum_{i=1}^{n} w_{i} x_{i} y_{i}
\end{aligned}
$$

and this delivers the desired conclusion.

The proof is complete.

Finally, we apply our results to get a refinement of the inequality of generalized arithmetic and geometric means.

Proposition 3.7. Assume $\left(C_{1}\right)$ and $\left(C_{2}\right)$. If $v_{1}, v_{2}, \ldots$ are positive numbers such that the series $\sum_{i=1}^{\infty} p_{i} v_{i}$ and $\sum_{i=1}^{\infty} p_{i} \ln \left(v_{i}\right)$ are absolutely convergent, then

$$
\sum_{i=1}^{\infty} p_{i} v_{i} \geq \prod_{i=1}^{\infty}\left(\frac{\sum_{j \in J} \lambda_{j} p_{\pi_{j}(i)} v_{\pi_{j}(i)}}{\sum_{j \in J} \lambda_{j} p_{\pi_{j}(i)}}\right)^{\sum_{j \in J} \lambda_{j} p_{\pi_{j}(i)}} \geq \prod_{i=1}^{\infty} v_{i}^{p_{i}}
$$

Proof. By applying Theorem 2.1 (b) to the convex function - ln, we obtain

$$
\begin{aligned}
& -\ln \left(\sum_{i=1}^{\infty} p_{i} v_{i}\right) \\
& \leq-\sum_{i=1}^{\infty}\left(\sum_{j \in J} \lambda_{j} p_{\pi_{j}(i)}\right) \ln \left(\frac{\sum_{j \in J} \lambda_{j} p_{\pi_{j}(i)} v_{\pi_{j}(i)}}{\sum_{j \in J} \lambda_{j} p_{\pi_{j}(i)}}\right) \leq-\sum_{i=1}^{\infty} p_{i} \ln \left(v_{i}\right),
\end{aligned}
$$

and this is equivalent to (3.7). 


\section{Acknowledgements}

Open access funding provided by University of Pannonia (PE). The research of the author has been supported by Hungarian National Foundations for Scientific Research Grant No. K120186.

Open Access. This article is licensed under a Creative Commons Attribution 4.0 International License, which permits use, sharing, adaptation, distribution and reproduction in any medium or format, as long as you give appropriate credit to the original author(s) and the source, provide a link to the Creative Commons licence, and indicate if changes were made. The images or other third party material in this article are included in the article's Creative Commons licence, unless indicated otherwise in a credit line to the material. If material is not included in the article's Creative Commons licence and your intended use is not permitted by statutory regulation or exceeds the permitted use, you will need to obtain permission directly from the copyright holder. To view a copy of this licence, visit http:// creativecommons.org/licenses/by/4.0/.

Publisher's Note Springer Nature remains neutral with regard to jurisdictional claims in published maps and institutional affiliations.

\section{References}

[1] Brnetić, I., Khan, K.A., Pečarić, J.: Refinement of Jensen's inequality with applications to cyclic mixed symmetric means and Cauchy means. J. Math. Inequal. 9(4), 1309-1321 (2015)

[2] Csiszár, I.: Information measures: a critical survey, Trans. 7th Prague Conference on Info. Th., Statist. Decis. Funct., Random Processes and 8th European Meeting of Statistics, Volume B, Academia Prague, pp. 73-86 (1978)

[3] Csiszár, I.: Information-type measures of difference of probability distributions and indirect observations. Studia Sci. Math. Hung. 2, 299-318 (1967)

[4] Csiszár, I., Körner, J.: Information Theory: Coding Theorems for Discrete Memoryless Systems. Academic Press, New York (1981)

[5] Dragomir, S.S.: A refinement of Jensen's inequality with applications for $f$-divergence measures. Taiwan. J. Math. 14, 153-164 (2010)

[6] Dragomir, S.S.: A new refinement of Jensen's inequality in linear spaces with applications. Math. Comput. Model. 52, 1497-1505 (2010)

[7] Horváth, L.: A refinement of the integral form of Jensen's inequality. J. Inequal. Appl. 2012, 178 (2012)

[8] Horváth, L., Khan, K.A., Pečarić, J.: Combinatorial improvements of Jensen's inequality. Classical and new refinements of Jensen's inequality with application. In: Monographs in Inequalities 8. Element, Zagreb, Croatia (2014)

[9] Horváth, L., Khan, K.A., Pečarić, J.: Cyclic refinements of the discrete and integral form of Jensen's inequality with applications. Analysis 36(4), 253-262 (2016)

[10] Horváth, L., Pečarić, D., Pečarić, J.: Estimations of $f$ - and Rényi divergences by using a cyclic refinement of the Jensen's inequality. Bull. Malays. Math. Sci. Soc. 42(3), 933-946 (2019)

[11] Niculescu, C., Persson, L.E.: Convex Functions and Their Applications. A Contemporary Approach. Springer, Berlin (2006)

[12] Pavić, Z.: Refinements of Jensen's inequality for infinite convex combinations. Turk. J. Inequal. 2(2), 44-53 (2018) 
[13] Perlman, M.D.: Jensen's inequality for a convex vector-valued function on an infinitedimensional space. J. Multivar. Anal. 4, 52-65 (1974)

\section{László Horváth}

Department of Mathematics

University of Pannonia

Egyetem u. 10.

Veszprém 8200

Hungary

e-mail: lhorvath@almos.uni-pannon.hu

Received: August 12, 2019

Revised: November 30, 2019 